\title{
Facilitated patent haemostasis after transradial catheterisation to reduce radial artery occlusion
}

\author{
Ahmad Edris ${ }^{*}$, MD; Jonathan Gordin², MD; Tamer Sallam², MD; Robin Wachsner ${ }^{3}$, MD; \\ Sheba Meymandi ${ }^{3}$, MD; Mahmoud Traina ${ }^{3}$, MD \\ 1. Cleveland Clinic Abu Dhabi, Abu Dhabi, UAE; 2. David Geffen School of Medicine at UCLA, Los Angeles, CA, USA; 3. Olive \\ View-UCLA Medical Center, Sylmar, CA, USA
}

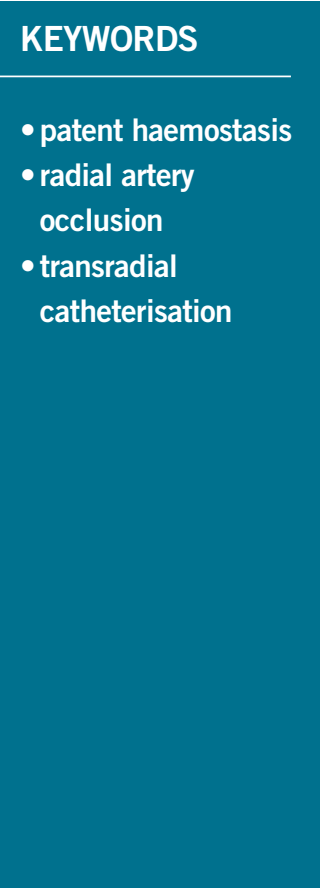

\begin{abstract}
Aims: This study sought to evaluate the feasibility of a rapid deflation technique (RDT) after transradial catheterisation to achieve patent haemostasis and to assess whether this could reduce radial artery occlusion (RAO). Ensuring patent haemostasis is the most important factor in reducing RAO. The use of larger sheath sizes and antiplatelet and antithrombotic agents limits achieving patent haemostasis immediately after transradial intervention.
\end{abstract}

Methods and results: A feasibility assessment was first performed in 105 patients to assess whether RDT could be performed safely and consistently achieve patent haemostasis after transradial catheterisation. Prospective data were then collected on 201 patients who underwent either rapid or standard deflation technique and had RAO assessment at 24 hours. Acute coronary syndrome was the indication for transradial catheterisation in $62.7 \%$ of patients. Baseline patent haemostasis increased from $40 \%$ to $95 \%$ after RDT. RAO at 24 hours was seen in two (2.0\%) patients in the RDT group and $15(14.9 \%)$ in the standard deflation group (OR 0.117; $95 \%$ CI: 0.026 to $0.526, \mathrm{p}=0.005$ ). Other independent predictors of RAO included body surface area (OR $0.022 ; 95 \%$ CI: 0.002 to 0.273 , $\mathrm{p}=0.003$ ) and male sex (OR $0.298 ; 95 \%$ CI: 0.108 to $0.824, \mathrm{p}=0.020$ ). No significant difference was found in safety outcomes: need to re-inflate compression band $(2 \%$ versus $1.8 \%)$ or haematoma $(0 \%$ versus $0.9 \%)$.

Conclusions: Rapid deflation of the compression band after transradial catheterisation is a safe and effective method of achieving patent haemostasis that reduces RAO.

*Corresponding author: Cleveland Clinic Abu Dhabi, Heart \& Vascular Institute, Sowwah Square, Al Maryah Island, PO Box 112412, Abu Dhabi, UAE.E-mail:edrisa@clevelandclinicabudhabi.ae 


\section{Introduction}

There is growing utilisation of transradial catheterisation for percutaneous angiography and intervention. The advantages of transradial over traditional femoral access include a decrease in the incidence of access-site bleeding complications and improvement in patient comfort and recovery ${ }^{1-3}$. However, transradial catheterisation is limited by radial artery occlusion (RAO). Although infrequent, and predominately asymptomatic in nature, RAO eliminates the radial artery access site from future catheterisation or use as an arterial conduit for surgical revascularisation or haemodialysis fistula. RAO is not always benign and hand ischaemia has been reported ${ }^{4-6}$. Consensus documents help define technical aspects required to optimise transradial catheterisation $^{7,8}$.

Editorial, see page 731

Previous studies have shown that increasing heparin dose $^{9}$, reducing post-procedure radial artery compression time ${ }^{10}$, and achieving post-procedure patent haemostasis ${ }^{11}$ decrease RAO rates. Ensuring patent haemostatic occlusion, the control of arterial bleeding while maintaining radial arterial flow, appears to be the single most important factor in reducing $\mathrm{RAO}^{12}$. Maintaining patent haemostasis has been shown to be feasible with minimal conversion to manual compression immediately after diagnostic catheterisation in low-risk, elective patients ${ }^{11}$. However, achieving patent haemostasis immediately after transradial intervention is limited by the use of larger sheath sizes and more potent antiplatelet and antithrombotic agents where bleeding limits the ability to lower the haemostatic pressure to achieve radial patency. We therefore sought to evaluate the feasibility of obtaining patent haemostasis at a fixed time interval after transradial catheterisation and interyention using a rapid deflation technique (RDT) in a predominately high-risk, acute coronary syndrome (ACS) patient population. After an initial feasibility study, we evaluated whether RDT could facilitate patent haemostasis and reduce the incidence of RAO.

\section{Methods}

All adult patients undergoing transradial catheterisation at Olive View-UCLA Medical Center (Sylmar, CA, USA) between July 2013 and September 2014 were considered for inclusion in the present study. The study was part of an institutional quality improvement initiative to reduce RAO at our centre by implementing standard of care consensus document recommendations ${ }^{8}$. A feasibility assessment was first performed in 105 patients to assess whether RDT could be performed safely and consistently achieve patent haemostasis. After the feasibility assessment, operators at our institution began to use either a standard or a rapid radial compression device deflation technique based on their preference. Prospective data were then collected on the next 201 consecutive patients based on whether they underwent operatordetermined standard compression band deflation or rapid deflation technique (RDT) 15 minutes after transradial catheterisation. The institutional review board of Olive View-UCLA Medical Center approved the study.
As part of an institute standard protocol, baseline patency of the ulno-palmar arch was ensured using a combination of pulse oximetry and plethysmography as described by Barbeau et $\mathrm{al}^{13}$. Patients who showed no recovery of pulse tracing within two minutes (response type D) were excluded from transradial access. Initial radial access, catheterisation, and sheath removal were similar in all patients following a catheterisation laboratory protocol. Patients were prepped and draped in a sterile fashion and $2 \%$ lidocaine was administered subcutaneously over the planned right radial artery puncture site. A 21-gauge, 1.5" (38 mm) metal needle was used to enter the radial artery $2-3 \mathrm{~cm}$ cranial to the bony prominence of the distal radius. A spring straight 0.021 " wire was then advanced into the radial artery. A GLIDESHEATH ${ }^{\mathrm{TM}}$ (Terumo Medical, Tokyo, Japan) was then advanced into the radial artery lumen using a modified Seldinger technique. In the feasibility portion, 5 Fr and 6 Fr sheaths were used, and, at the time of RAO outcomes assessment, only $6 \mathrm{Fr}$ sheaths were employed. A combination of nitroglycerine $200 \mathrm{mcg}$, verapamil $2.5 \mathrm{mg}$, and unfractionated heparin 50 units $/ \mathrm{kg}$ (maximum 5,000 units) was administered into the radial artery unless prohibited by hypotension. Coronary angiography was performed using 5 Fr diagnostic catheters and interventions using 6 Fr guide catheters. Weight-based unfractionated heparin alone or bivalirudin $0.75 \mathrm{mg} / \mathrm{kg}$ bolus followed by $1.75 \mathrm{mg} / \mathrm{kg} / \mathrm{hr}$ drip was used if percutaneous coronary intervention was performed. Activated clotting time was checked to ensure a time above 250 seconds in patients undergoing intervention. In all patients undergoing stent placement, aspirin $(325 \mathrm{mg}$ ) and clopidogrel $(600 \mathrm{mg})$ were given at the time of catheterisation unless previously received.

Immediately after the procedure, the radial artery introducer sheath was withdrawn $2-3 \mathrm{~cm}$ and a radial compression TR Band $^{\mathrm{TM}}$ (Terumo Medical) device applied according to standard TR Band manufacturer protocol: the air bladder of the TR Band was filled with $18 \mathrm{~mL}$ of air to achieve initial haemostasis. The sheath was then removed and the air bladder slowly deflated until bleeding was visualised. Then, $2 \mathrm{~mL}$ of air was reintroduced into the bladder to achieve haemostasis.

\section{FEASIBILITY ASSESSMENT}

Exactly 15 minutes after placement of the TR Band, patency of the radial artery was assessed in 105 consecutive patients in order to establish a baseline patent haemostasis rate. Patency was assessed using plethysmography and pulse oximetery while the ipsilateral ulnar artery was occluded (reverse Barbeau). Radial artery patency was documented if present; otherwise, the TR Band was slowly deflated until plethysmographic signal returned in order to restore radial patency. The compression balloon was then further deflated until either bleeding occurred or a minimum balloon volume of $7 \mathrm{~mL}$ was achieved. The compression balloon was then reflated $2 \mathrm{~mL}$ above the bleeding point or left with a minimum of $7 \mathrm{~mL}$ of air. The TR Band remained in place for two hours and was then removed. Although bleeding was evaluated, an assessment of RAO was not performed during the feasibility assessment. 


\section{ASSESSMENT OF RADIAL ARTERY OCCLUSION}

After the feasibility assessment, operators at our institution began to employ one or other of two different TR Band deflation strategies:

1. Standard deflation technique (SDT): TR Band applied at the end of the procedure according to standard TR Band manufacturer protocol described previously. Patent haemostasis was not assessed and TR Band maintained for two hours and removed.

2. Rapid deflation technique (RDT): exactly 15 minutes after TR Band application, radial artery patency was evaluated using a reverse Barbeau test with occlusion of the ulnar artery. The TR Band was then deflated to the lowest allowable volume (minimum $7 \mathrm{~mL}$ ) while maintaining haemostasis. If bleeding occurred, $2 \mathrm{~mL}$ of air was reintroduced from the bleeding point. Final radial artery patency was documented if present and the TR Band was then maintained for two hours before being removed.

Prospective data were collected on 201 non-randomised patients who underwent one or other deflation technique based on operator preference. Patients were included in the analysis only if an assessment of radial artery occlusion at 24 hours was performed. Patient characteristics, history, and laboratory results were obtained via a review of the electronic medical record (EMR). Procedural characteristics were obtained on review of the catheterisation laboratory electronic logs. Unstable angina was defined as progressive symptoms of angina with or without electrocardiogram (ECG) changes but without positive cardiac biomarkers. Non-STelevation myocardial infarction (NSTEMI) and ST-elevation myocardial infarction (STEMI) were defined in accordance with the universal definition of myocardial infarction ${ }^{14}$.

The primary outcome, radial artery patency, was evaluated at 24 hours using a reverse Barbeau test utilising plethysmographic and pulse oximetry evaluation. Safety outcomes included the need to reinflate the TR Band due to bleeding or haematoma formation at the access site.

\section{STATISTICAL ANALYSIS}

Data management and analysis were performed with SPSS Statistics, Version 22 (IBM Corp., Armonk, NY, USA). Continuous variables are presented as mean $\pm \mathrm{SD}$ and means are compared with the Student's t-test. Categorical variables are given as proportions and compared with the chi-square or Fisher's exact test. All statistical tests are two-sided and statistical significance defined as a p-value $<0.05$. Univariate associations with radial artery occlusion were tested for all patient and procedural characteristics. All univariate predictors with a $\mathrm{p}$-value $<0.15$ along with predetermined predictors (sex, body surface area, duration of sheath [min], initial TR Band air $[\mathrm{mL}]$, stent placement) were included in a multivariate model. The predictors were then removed in a stepwise fashion, starting with the highest $\mathrm{p}$-value until only significant factors remained.

\section{Results}

A total of 105 patients were enrolled in the initial feasibility study. The majority (58\%) had 6 Fr sheaths used for radial artery access.
Percutaneous coronary intervention (PCI) was performed in $26 \%$ of the initial feasibility study patients. In those undergoing PCI, $70 \%$ received bivalirudin. Clopidogrel pretreatment was used in $36 \%$ of patients.

Patent haemostasis was found to be present in $15 \%(n=16)$ of patients in the feasibility assessment performed 15 minutes after placement of the TR Band. The volume of air in the TR Band post procedure was similar between the group of patients without initial patent haemostasis and the patent haemostasis group, $13.45 \mathrm{~mL}$ and $13.38 \mathrm{~mL}$, respectively. In the group without initial patent haemostasis, the TR Band had to be deflated an average $3.51 \mathrm{~mL}$ before radial artery patency was restored. The final average TR Band volume was similar in both groups, $7.40 \mathrm{~mL}$ for the patients without initial patent haemostasis and $7.56 \mathrm{~mL}$ for the other group (Figure 1). No bleeding complications were found, and all patients were able to achieve patent haemostasis after initial evaluation and TR Band deflation.

In the assessment of radial artery occlusion study, data were reviewed on 201 patients who underwent one or other of two strategies: standard deflation or rapid deflation technique after TR Band placement. The baseline characteristics were similar between the two groups (Table 1), including percentage of males, mean body surface area, and other anticoagulant use. Patients in the RDT group were older $(58.6 \pm 10.3$ versus $56.1 \pm 8.1$ years, $\mathrm{p}=0.05$ ), had a higher frequency of prior known coronary artery disease (CAD) (25\% versus $15 \%, \mathrm{p}=0.079)$, and of prior percutaneous coronary intervention (PCI) (18\% versus $7 \%, \mathrm{p}=0.019)$. Risk factors for atherosclerotic disease, including diabetes mellitus, hypertension, dyslipidaemia, and tobacco use were highly prevalent in both groups. Acute coronary syndrome (ACS) was the indication for the procedure in $62.7 \%$ of patients. Rates of guideline-based, pre-procedural medications for ACS were similar in both groups, with $87.2 \%$ of patients receiving aspirin and $38.9 \%$ receiving clopidogrel at least two hours before catheterisation.

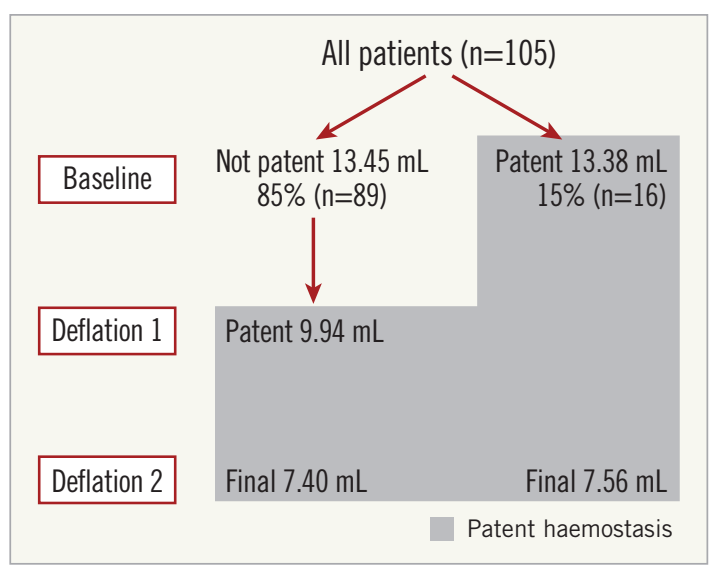

Figure 1. Feasibility study: TR Band volume and patent haemostasis. Radial artery patency evaluated at baseline. Patients with radial patency at baseline $(n=16)$ underwent single deflation. Mean TR Band volumes reported. 
Table 1. Radial artery occlusion study: patient demographics. Characteristic SDT ( $n=101)$ RDT ( $n=100) \mid p$-value

\begin{tabular}{|c|c|c|c|c|}
\hline \multicolumn{2}{|c|}{ Age (years) } & $56.1 \pm 10.3$ & $58.6 \pm 8.1$ & 0.05 \\
\hline \multicolumn{2}{|c|}{ Sex (males \%) } & 63.4 & 72.0 & NS \\
\hline \multicolumn{2}{|l|}{$\mathrm{BSA}\left(\mathrm{m}^{2}\right)$} & $1.87 \pm 0.26$ & $1.92 \pm 0.27$ & NS \\
\hline \multicolumn{2}{|c|}{ Diabetes mellitus (\%) } & 51.5 & 44.0 & NS \\
\hline \multicolumn{2}{|c|}{ Hypertension (\%) } & 78.2 & 70.0 & NS \\
\hline \multicolumn{2}{|c|}{ Hyperlipidaemia (\%) } & 49.5 & 51.0 & NS \\
\hline \multicolumn{2}{|c|}{ Known CAD (\%) } & 15.0 & 25.0 & NS \\
\hline \multicolumn{2}{|c|}{ Prior PCl (\%) } & 7.0 & 18.0 & 0.019 \\
\hline \multicolumn{2}{|c|}{ Prior CABG (\%) } & 1.0 & 1.0 & NS \\
\hline \multirow{2}{*}{$\begin{array}{l}\text { Tobacco } \\
\text { use }\end{array}$} & Current (\%) & 25.7 & 23.0 & NS \\
\hline & Prior (\%) & 30.7 & 35.0 & NS \\
\hline \multicolumn{2}{|c|}{ INR (mean) } & $1.17 \pm 0.36$ & $1.13 \pm 0.27$ & NS \\
\hline \multicolumn{2}{|c|}{ Pre-procedure aspirin (\%) } & 82.2 & 92.2 & NS \\
\hline \multicolumn{2}{|c|}{ Pre-procedure clopidogrel (\%) } & 34.7 & 43.0 & NS \\
\hline
\end{tabular}

*Plus-minus values are means \pm SD. RDT: rapid deflation technique; SDT: standard deflation technique

Procedural characteristics were also similar between the SDT and RDT groups (Table 2). As previously noted, at the time of the RAO assessment study, all patients received 6 Fr sheaths. There was no significant difference in the duration of sheath insertion or the use of an antithrombotic agent such as heparin or bivalrudin in addition to the heparin administered upon initial sheath insertion. Although there was a trend towards a greater volume of air in the TR Band in the SDT group $(13.5 \pm 2.2 \mathrm{~mL}$ versus $14 \pm 2 \mathrm{~mL}$ in the SDT group, $\mathrm{p}=0.068$ ), the absolute difference was minimal at $0.5 \mathrm{~mL}$.

Table 2. Radial artery occlusion study: procedural characteristics.

\begin{tabular}{|c|c|c|c|}
\hline Characteristic & SDT $(n=101)$ & RDT $(n=100)$ & $p$-value \\
\hline Stable angina (\%) & 7.0 & 6.0 & NS \\
\hline Unstable angina (\%) & 42.6 & 42.0 & NS \\
\hline NSTEMI (\%) & 19.8 & 20.0 & NS \\
\hline STEMI (\%) & 1.0 & 0 & NS \\
\hline Sheath duration $(\mathrm{min})^{*}$ & $48.1 \pm 34.2$ & $46.1 \pm 36.0$ & NS \\
\hline Total catheters* & $2.66 \pm 0.87$ & $2.49 \pm 0.64$ & NS \\
\hline Procedural anticoagulation (\%) & 39.6 & 38.3 & NS \\
\hline Heparin (\%) & 19.8 & 12.0 & NS \\
\hline Bivalrudin (\%) & 19.8 & 27.0 & NS \\
\hline Intervention (\%) & 26.7 & 36.0 & NS \\
\hline Initial TR Band volume $(\mathrm{mL})$ * & $14.0 \pm 2.0$ & $13.5 \pm 2.2$ & NS \\
\hline Initial patent haemostasis (\%) & $N / A$ & 40.0 & $\mathrm{~N} / \mathrm{A}$ \\
\hline New TR Band volume $(\mathrm{mL})^{*}$ & $\mathrm{~N} / \mathrm{A}$ & $8.1 \pm 2.0$ & $\mathrm{~N} / \mathrm{A}$ \\
\hline New patent haemostasis (\%) & $\mathrm{N} / \mathrm{A}$ & 95.0 & $\mathrm{~N} / \mathrm{A}$ \\
\hline
\end{tabular}

*means \pm SD. NSTEMI: non-ST-elevation myocardial infarction; RDT: rapid deflation technique; SDT: standard deflation technique; STEMI: ST-elevation myocardial infarction
Patent haemostasis was found to be present at baseline in $40.0 \%$ of patients in the RDT group. Patent haemostasis increased to 95.0\% after RDT and lowering the TR Band volume from an average $13.5 \mathrm{~mL}$ to $8.1 \mathrm{~mL}$ (Table 2). RAO at 24 hours was seen in two $(2.0 \%)$ patients in the RDT group and in 15 (14.9\%) patients in the SDT group (Figure 2, Table 3), which was highly significant $(\mathrm{p}=0.002)$. In multivariate analysis using a logistic regression model, deflation technique remained an independent predictor of RAO with an odds ratio of 0.117 (95\% CI: 0.026 to 0.526 , $\mathrm{p}=0.005)$ for the rapid deflation technique. In the same model, body surface area had an odds ratio of 0.022 (95\% CI: 0.002 to $0.273, \mathrm{p}=0.003)$ and male sex had an odds ratio of 0.298 (95\% CI: 0.108 to $0.824, \mathrm{p}=0.020$ ). In terms of safety outcomes, there was no significant difference in TR Band reinflation in the RDT group compared to the SDT group ( $2 \%$ versus $1.8 \%$ ), or in the incidence of haematoma ( $0 \%$ versus $0.9 \%$ ) (Figure 3, Table 3 ).

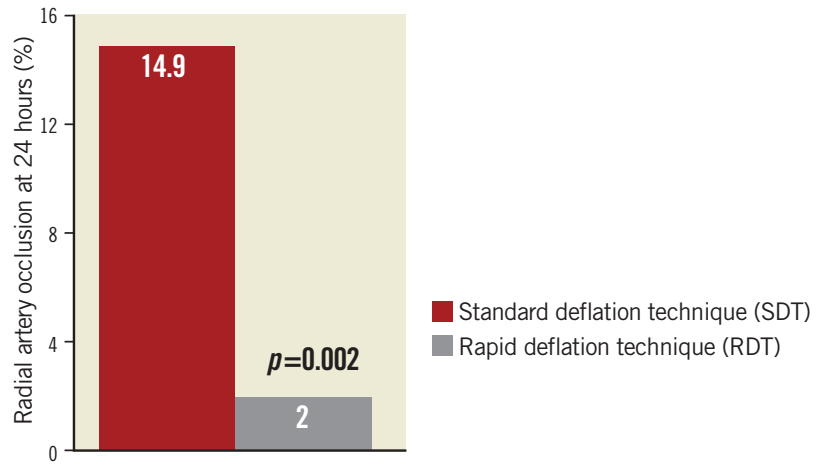

Figure 2. Radial artery occlusion study: rate of occlusion at 24 hours.

Table 3. Radial artery occlusion study: outcomes.

\begin{tabular}{|l|c|c|c|}
\hline \multicolumn{1}{|c|}{ Characteristic } & SDT $(\boldsymbol{n}=101)$ & RDT $(\boldsymbol{n}=100)$ & $\boldsymbol{p}$-value \\
\hline Need to reinflate (\%) & 1.8 & 2.0 & NS \\
\hline Haematoma (\%) & 0.9 & 0 & NS \\
\hline Radial artery occlusion (\%) & 14.9 & 2.0 & 0.002 \\
\hline RDT: rapid deflation technique; SDT: standard deflation technique \\
\hline
\end{tabular}

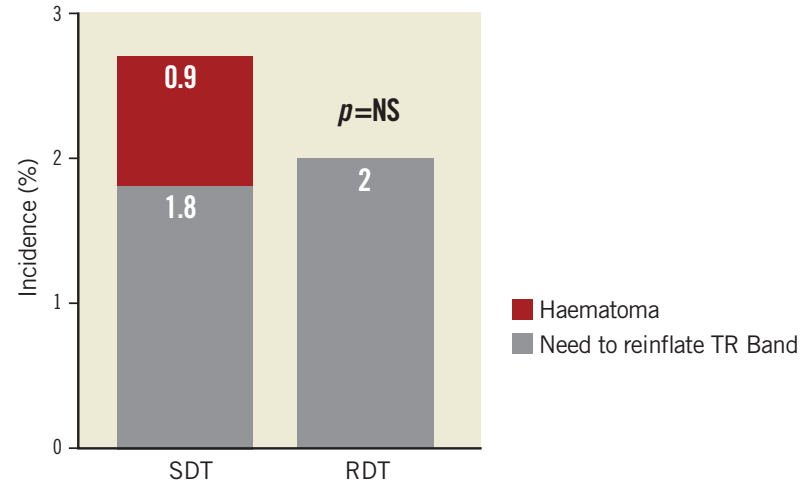

Figure 3. Radial artery occlusion study: safety outcomes. RDT: rapid deflation technique; SDT: standard deflation technique 


\section{Discussion}

Among patients undergoing transradial catheterisation and intervention, patent haemostasis was found to be present in the minority of patients using standard TR Band compression balloon application. By waiting a specified 15 minutes after placement of the TR Band, we found that the compression balloon could be safely deflated achieving patent haemostasis in almost all patients without bleeding complications. This single, rapid deflation technique (RDT) was associated with a significant reduction in radial artery occlusion at 24 hours as compared to standard TR Band application and deflation at two hours.

Although achieving patent haemostasis has been shown in low-risk patients using 5 Fr sheaths with minimal conversion to manual compression ${ }^{11}$, the feasibility of patent haemostasis in high-risk ACS patients using larger 6 Fr sheaths has not been studied. Many operators have found it difficult to achieve patent haemostasis immediately after transradial intervention when larger sheaths are employed and patients are fully anticoagulated. This study included a large proportion of patients with ACS receiving 6 Fr sheaths. Despite the increased risk of bleeding in this group of patients, we found that the TR Band could be safely deflated significantly below the patent haemostasis point without bleeding complications by waiting a specified 15 minutes after placement of the compression device. Initial formation of the platelet plug and thrombosis of the tissue tract may explain the lower TR Band volume required at 15 minutes to maintain haemostasis. The rate of patent haemostasis before rapid deflation was $15 \%$ in the feasibility study and $40 \%$ in the RAO assessment despite a standard TR Band manufacturer protocol that used a minimum of haemostatic air pressure to achieve haemostasis after sheath removal. Patent haemostasis increased significantly after RDT to $100 \%$ in the feasibility study and $95 \%$ in the RAO assessment portion. The higher initial radial artery patency in the RAO assessment study may be explained by the increased frequency of intervention performed in this part of the trial and the exposure to additional anticoagulation beyond the initial heparin (intervention rate: $26 \%$ in feasibility trial versus $31 \%$ in RAO assessment).

In the second portion of our trial, we evaluated whether RDT as compared to standard deflation was associated with a reduction in the rate of RAO. Despite being a non-randomised trial, the two groups were very well matched with a significant proportion being ACS patients (62.7\%) exposed to antiplatelet and antithrombotic medications. All patients received 6 Fr sheaths. Radial artery occlusion was assessed at 24 hours using a clinically relevant and simple reverse Barbeau test utilising plethysmographic and pulse oximetry evaluation. The RAO rate was noted to be $2 \%$ in the rapid deflation group compared to $14.9 \%$ using a standard deflation technique. The rate of RAO in the standard deflation group was higher than expected from previous data. Prior trials have reported RAO rates at 24 hours between $4.3 \%$ and $12 \%{ }^{9-12,15,16}$. However, the majority of these trials used 5 Fr sheaths; a higher rate of RAO has been established with the use of a larger sheath size $^{17}$. In fact, Uhlemann et al reported a significant increase in the rate of radial occlusion at 24 hours in patients receiving 6 Fr sheaths as compared to 5 Fr sheaths (30.5\% versus $13.7 \%$, $\mathrm{p}<0.001)^{18}$.

This trial did not look at TR Band compression pressure but rather a surrogate that was more clinically applicable and measurable, TR Band air volume. The deflation of the TR Band at 15 minutes reduces arterial compressive pressure and allows patent haemostasis, as evidenced by the return of plethysmographic signal during reverse Barbeau evaluation. The TR Band compressive pressure and duration of application have a direct effect on patent haemostasis: radial artery patency is decreased and RAO increased if the TR Band is not deflated at some point after transradial catheterisation. Some institutions employ protocols that call for a gradual deflation of the TR Band over time. An argument could be made that our standard therapy group had the band inflated too long at the initial volume. However, gradual deflation techniques are labour-intensive at the nursing level and require frequent workflow interruptions. Further, our study found that the TR Band needed to be deflated an average of $3.51 \mathrm{~mL}$, and in some cases up to $9 \mathrm{~mL}$, to achieve patent haemostasis. A gradual deflation protocol would delay the onset of patent haemostasis, and could potentially jeopardise radial artery patency. The RDT allows a simple, single band adjustment 15 minutes after the placement of the TR Band and can be performed almost always before the patient is transported to recovery.

\section{Study limitations}

The present study has several important limitations. Primarily, there was no randomisation: two different institution operators employed the different techniques when they were performing catheterisation. This raises a concern about the possible effect of the operator on the observed outcome. However, this potential effect should be minimal given that the same sheath removal and TR Band placement procedure was used in all patients according to a standard catheterisation lab protocol. Additionally, procedural characteristics were well balanced in the two groups. Although there were 201 patients in the RAO assessment portion of the study, given the large difference in outcomes between the groups and the small number of occlusions in the RDT group, our confidence interval for the primary outcome is large. The statistical significance of the deflation technique, even after multivariate analysis, remains valid. Finally, RAO was assessed at 24 hours only; prior trials have shown that a significant proportion (36$58 \%$ ) of patients will have spontaneous resolution of radial occlusion at 30 days $^{10-12,15}$. Despite this fact, the projected rate of RAO beyond 30 days remains high and predominantly unidentified until the patient requires repeat transradial catheterisation or the radial artery is considered for surgical grafting.

\section{Conclusions}

We conclude that routinely lowering the TR Band to the lowest permissible volume while still maintaining haemostasis $15 \mathrm{~min}$ utes after transradial catheterisation is a safe and effective method 
of achieving patent haemostasis in almost all patients. This technique is not only feasible in patients with larger sheath sizes and more potent anticoagulation but also associated with a significant reduction in RAO without bleeding complications or the need to reinflate the radial compression device. Further, prospectively randomised studies should be conducted to confirm these findings and compare this method to other deflation techniques as well as to monitor other possible effects, such as patient satisfaction and pain.

\section{Impact on daily practice}

There are several TR Band deflation and removal protocols that are often difficult to implement at a physician or nursing level, with no standardisation. We propose a simplification of TR Band management; a single deflation of the TR Band to the lowest allowable volume (minimum $7 \mathrm{~mL}$ ) while maintaining haemostasis, performed 15 minutes after sheath removal and application of the haemostatic compression device, followed by complete removal after two hours. This rapid deflation technique is safe and associated with a reduction in RAO, and may both facilitate patient comfort and simplify the process for the cardiac catheterisation and nursing team.

\section{Conflict of interest statement}

The authors have no conflicts of interest to declare.

\section{References}

1. Pristipino C, Pelliccia F, Granatelli A, Pasceri V, Roncella A, Speciale G, Hassan T, Richichi G. Comparison of access-related major bleeding complications in women versus men undergoing percutaneous coronary catheterization using the radial versus femoral artery. Am J Cardiol. 2007;99:1216-21.

2. Jolly SS, Amlani S, Hamon M, Yusuf S, Mehta SR. Radial versus femoral access for coronary angiography or intervention and the impact on major bleeding and ischemic events: a systematic review and meta-analysis of randomized trials. Am Heart J. 2009;157:132-40.

3. Jolly SS, Yusuf S, Cairns J, Niemelä K, Xavier D, Widimsky P, Budaj A, Niemelä M, Valentin V, Lewis BS, Avezum A, Steg PG, Rao SV, Gao P, Afzal R, Joyner CD, Chrolavicius S, Mehta SR; RIVAL trial group. Radial versus femoral access for coronary angiography and intervention in patients with acute coronary syndrome (RIVAL): a randomised, parallel group, multicentre trial. Lancet. 2011;377:1409-22.

4. Rhyne D, Mann T. Hand ischemia resulting from a transradial intervention: successful management with radial artery angioplasty. Catheter Cardiovasc Interv. 2010;76:383-6.

5. Ruzsa Z, Pinter L, Kolvenbach R. Anterograde recanalisation of the radial artery followed by transradial angioplasty. Cardiovasc Revasc Med. 2010;11:266.

6. Greenwood MJ, Della-Siega AJ, Fretz EB, Kinloch D, Klinke P, Mildenberger R, Williams MB, Hilton D. Vascular communications of the hand in patients being considered for transradial coronary angiography: is the Allen's test accurate? $\mathrm{J} \mathrm{Am}$ Coll Cardiol. 2005;46:2013-7.

7. Hamon M, Pristipino C, Di Mario C, Nolan J, Ludwig J, Tubaro M, Sabate M, Mauri-Ferre J, Huber K, Niemela K, Haude M, Wijns W, Dudek D, Fajadet J, Kiemeneij F; European Association of Percutaneous Cardiovascular Interventions; Working Group on Acute Cardiac Care of the European Society of Cardiology; Working Group on Thrombosis on the European Society of Cardiology. Consensus document on the radial approach in percutaneous cardiovascular interventions: position paper by the European Association of Percutaneous Cardiovascular Interventions and Working Groups on Acute Cardiac Care and Thrombosis of the European Society of Cardiology. EuroIntervention. 2013;8: 1242-51.

8. Rao SV, Tremmel JA, Gilchrist IC, Shah PB, Woody W, Zoghbi G, Duffy PL, Sanghvi K, Krucoff MW, Pyne CT, Skelding KA, Patel T, Pancholy SB; Society for Cardiovascular Angiography and Intervention's Transradial Working Group. Best practices for transradial angiography and intervention: a consensus statement from the society for cardiovascular angiography and intervention's transradial working group. Catheter Cardiovasc Interv. 2014;83:228-36.

9. Spaulding C, Lefèvre T, Funck F, Thébault B, Chauveau M, Ben Hamda K, Chalet Y, Monségu J, Tsocanakis O, Py A, Guillard N, Weber S. Left radial approach for coronary angiography: results of a prospective study. Cathet Cardiovasc Diagn. 1996; 39:365-70.

10. Pancholy SB, Patel TM. Effect of duration of hemostatic compression on radial artery occlusion after transradial access. Catheter Cardiovasc Interv. 2012;79:78-81.

11. Pancholy S, Coppola J, Patel TM, Roke-Thomas M. Prevention of radial artery occlusion: patent hemostasis evaluation trial: a randomized comparison of traditional versus patency documented hemostasis after transradial catheterization. Catheter Cardiovasc Interv. 2008;72:335-40.

12. Pancholy SB, Bertrans OF, Patel TM. Comparison of a priori versus provisional heparin therapy on radial artery occlusion after transradial coronary angiography and patent hemostasis (from the PHARAOH Study). Am J Cardiol. 2012;110:173-6.

13. Barbeau GR, Arsenault F, Dugas L, Simard S, Larivière MM. Evaluation of the ulnopalmar arterial arches with pulse oximetry and plethysmography: comparison with the Allen's test in 1010 patients. Am Heart J. 2004;147:489-93.

14. Thygesen K, Alpert JS, Jaffe AS, Simoons ML, Chaitman BR, White HD; Joint ESC/ACCF/AHA/WHF Task Force for Universal Definition of Myocardial Infarction; Authors/Task Force Members Chairpersons, Thygesen K, Alpert JS, White HD; Biomarker Subcommittee, Jaffe AS, Katus HA, Apple FS, Lindahl B, Morrow DA; ECG Subcommittee, Chaitman BR, Clemmensen PM, Johanson P, Hod H; Imaging Subcommittee, Underwood R, Bax JJ, Bonow JJ, Pinto F, Gibbons RJ; Classification Subcommittee, Fox KA, Atar D, Newby LK, Galvani M, Hamm CW; Intervention 
Subcommittee, Uretsky BF, Steg PG, Wijns W, Bassand JP, Menasche P, Ravkilde J; Trials \& Registries Subcommittee, Ohman EM, Antman EM, Wallentin LC, Armstrong PW, Simoons ML; Trials \& Registries Subcommittee, Januzzi JL, Nieminen MS, Gheorghiade M, Filippatos G; Trials \& Registries Subcommittee, Luepker RV, Fortmann SP, Rosamond WD, Levy D, Wood D; Trials \& Registries Subcommittee, Smith SC, Hu D, Lopez-Sendon JL, Robertson RM, Weaver D, Tendera M, Bove AA, Parkhomenko AN, Vasilieva EJ, Mendis S; ESC Committee for Practice Guidelines (CPG), Bax JJ, Baumgartner H, Ceconi C, Dean V, Deaton C, Fagard R, Funck-Brentano C, Hasdai D, Hoes A, Kirchhof P, Knuuti J, Kolh P, McDonagh T, Moulin C, Popescu BA, Reiner Z, Sechtem U, Sirnes PA, Tendera M, Torbicki A, Vahanian A, Windecker S; Document Reviewers, Morais J, Aguiar C, Almahmeed W, Arnar DO, Barili F, Bloch KD, Bolger AF, Botker HE, Bozkurt B, Bugiardini R, Cannon C, de Lemos J, Eberli FR, Escobar E, Hlatky M, James S, Kern KB, Moliterno DJ, Mueller C, Neskovic AN, Pieske BM, Schulman SP, Storey RF,
Taubert KA, Vranckx P, Wagner DR. Third universal definition of myocardial infarction. J Am Coll Cardiol. 2012;60:1581-98.

15. Pancholy MB. Comparison of the effect of intra-arterial versus intravenous heparin on radial artery occlusion after transradial catheterization. Am J Cardiol. 2009;104:1083-5.

16. Dharma S, Kedev S, Patel T, Kiemeneij F, Gilchrist IC. A novel approach to reduce radial artery occlusion after transradial catheterization: postprocedural/prehemostasis intra-arterial nitroglycerin. Catheter Cardiovasc Interv. 2015;85:818-25.

17. Saito S, Ikei H, Hosokawa G, Tanaka S. Influence of the ratio between radial artery inner diameter and sheath outer diameter on radial artery flow after transradial coronary intervention. Catheter Cardiovasc Interv. 1999;46:173-8.

18. Uhlemann M, Möbius-Winkler S, Mende M, Eitel I, Fuernau G, Sandri M, Adams V, Thiele H, Linke A, Schuler G, Gielen S. The Leipzig prospective vascular ultrasound registry in radial artery catheterization: impact of sheath size on vascular complications. JACC Cardiovasc Interv. 2012;5:36-43. 